\title{
Plasma condensation in coronal loops ${ }^{\star}$
}

\author{
E. O'Shea ${ }^{1}$, D. Banerjee ${ }^{2}$, and J. G. Doyle ${ }^{1}$ \\ 1 Armagh Observatory, College Hill, Armagh BT61 9DG, N. Ireland \\ e-mail: eos@arm.ac.uk \\ 2 Indian Institute of Astrophysics, II Block, Koramangala, Bangalore 560 034, India
}

Received 5 September 2007 / Accepted 2 October 2007

\begin{abstract}
Aims. Using temporal series data from the Coronal Diagnostic Spectrometer (CDS) on SOHO, we seek to investigate plasma condensation through the use of spectroscopic analysis.

Methods. Using a space-time (X-T) type plot we show, for a number of transition region (TR) and coronal lines, the variation of intensity (radiant flux) with time in an off-limb loop system. Noting a number of pixels where the intensity shows a sharp jump in value for the TR lines and a corresponding sharp decrease for the coronal lines, we investigate further and plot the intensity and velocity for these pixels as a function of time. Using standard Fourier techniques, together with a pre-whitening method, we measure all statistically significant frequencies present in the time series so created.

Results. We find that, for the cooler lines of He I $584 \AA$ and O V $629 \AA$, there are sharp increases in intensity, accompanied by large blueshifted velocities. In the case of $\mathrm{OV}$ there is a substantial rebound to redshifted velocities when the strong brightening fades. The sharp increase in intensity and blueshifted velocity in the cooler lines is accompanied by a corresponding decrease in the intensity of the coronal lines (Mg X $624 \AA$, Fe XVI $360 \AA$, Si XII $520 \AA$ ), but with no corresponding change in their velocity values implying an evacuation of plasma. From the Fourier analysis, evidence is found for possible 1st and 2 nd harmonics in the He I and O v lines, indicative of standing waves in loops.

Conclusions. We conclude that what we are seeing is the first spectroscopic evidence of plasma condensation taking place in coronal loops.
\end{abstract}

Key words. Sun: UV radiation - Sun: atmosphere - Sun: transition region - Sun: corona - Sun: oscillations

\section{Introduction}

Recent observations have revealed that magnetically closed structures in the upper solar atmosphere, commonly referred to as coronal loops, exhibit intrinsically dynamic behavior (De Groof et al. 2004; Doyle et al. 2006; O’Shea et al. 2007). Even in quiescent, non-flaring conditions, loops show strong temporal variability of emission in UV spectral lines and substantial plasma flows. Spectroscopic investigations show that these intensity variations have different signatures in UV spectral lines formed at different temperatures and exhibit Doppler shifts of $v \approx 20-130 \mathrm{~km} \mathrm{~s}^{-1}$ (Fredvik et al. 2002; Teriaca et al. 2004). In an overview of observations of the temporal variability of active region loops with the Coronal Diagnostic Spectrometer (CDS), Kjeldseth-Moe \& Brekke (1998) report significant changes of coronal loops over a period of one hour, in particular seen in emission lines in the $1-5 \times 10^{5} \mathrm{~K}$ temperature range. This variability is accompanied by large Doppler shifts, typically around $v=50-100 \mathrm{~km} \mathrm{~s}^{-1}$. Observations with the Extreme-ultraviolet Imaging Telescope (EIT) with high temporal cadence (De Groof et al. 2004) furthermore reveal spatially localized brightenings in coronal loops, moving rapidly down towards the footpoints of the loops. The fact that coronal loops can undergo rapid evacuation has been known for decades. For example, Levine \& Withbroe (1977) reported on Skylab spectroscopic observations that were compatible with a "dramatic

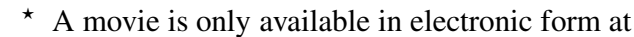
http: //www . aanda.org evacuation" of active region loops triggered by rapid, radiation dominated cooling. A detailed study of "catastrophic cooling" and evacuation of quiescent coronal loops observed with the TRACE instrument is presented by Schrijver (2001). He analysed image sequences taken in different spectral passbands and found that loop evacuation occurs frequently after plasma in the upper parts of the loops has cooled to transition region (TR) or lower temperatures. The cooling process is often accompanied by emission in Ly $\alpha$ and C IV (1548 $\AA$ ), developing initially near the loop top. Thereafter, cool plasma is observed to slide down on both sides of the loop, forming clumps which move with velocities of up to $100 \mathrm{~km} \mathrm{~s}^{-1}$. The downward acceleration of these plasma clumps as inferred from these observations is significantly less than the gravitational acceleration on the solar surface. According to the observations of Schrijver (2001), this process of dramatic cooling and evacuation is a rather common one. Further observational evidence of "blobs" of plasma falling down towards the solar surface along magnetic field lines is presented by De Groof et al. (2005), based on high cadence time series of simultaneous EIT (304 $\AA$ ) and Big Bear data. In this paper we will report on the spectroscopic detection of plasma condensation as seen by CDS on SOHO.

\section{Observations and data reduction}

To obtain the main part of our observations, we used the Normal Incidence Spectrometer (NIS), part of the Coronal Diagnostic Spectrometer (CDS) on board the Solar and Heliospheric 

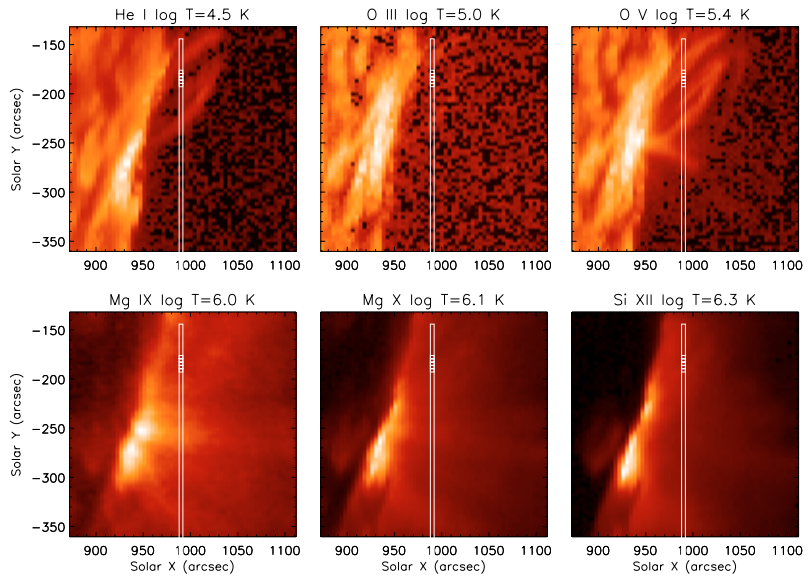

Fig. 1. Raster images from dataset s27153r00. The vertical lines show the location of the CDS slit, corresponding to the temporal series dataset s27152r01 and the small white boxes (between $Y \approx-200 \rightarrow$ $\left.-170^{\prime \prime}\right)$ mark the location of the pixels 55-59.

Observatory (SOHO) (Harrison et al. 1995). For context movies, we also made use of TRACE (Handy et al. 1999) $171 \AA$ data.

The temporal sequence OS_AR85 (dataset 27152r01) was run in active region AR10314 at the Western limb of the Sun between 10:32-11:01 UTC on the 21st March 2003. This was a CDS test run for the joint observing campaign, JOP165 ${ }^{1}$, that was first run on the 27th March 2003. During this test run no EIT (shutterless) observations were obtained. Data were obtained using slit No. $5\left(4^{\prime \prime} \times 240^{\prime \prime}\right)$ for 6 TR and coronal lines; He I $584.33 \AA\left(\approx 3 \times 10^{4} \mathrm{~K}\right), \mathrm{O}$ III $599.59 \AA(\approx 1 \times$ $\left.10^{5} \mathrm{~K}\right), \mathrm{O}$ V $629.73 \AA\left(\approx 2.5 \times 10^{5} \mathrm{~K}\right)$ and the coronal lines of $\mathrm{Mg}$ X $624.94 \AA\left(\approx 1.25 \times 10^{6} \mathrm{~K}\right)$, Si XII $520.67 \AA(\approx 2 \times$ $\left.10^{6} \mathrm{~K}\right)$ and Fe XVI $360.76 \AA\left(\approx 2.5 \times 10^{6} \mathrm{~K}\right)$. Binning by 2 along the 143 pixel slit onboard SOHO resulted in 70 usable pixels of size $4^{\prime \prime}$ (in $X$ ) $\times 3.36^{\prime \prime}$ (in $Y$ ), where $Y$ is the north-south direction. A context raster sequence, GENE/v97 (dataset s27153r00) was also run on the 21st March 2003 between the times of 11:50-12:12 UTC, $\approx 50 \mathrm{~min}$ after the end of the OS_AR85 sequence. This raster sequence contains the same lines as OS_AR85, with the addition of Mg IX $368.06 \AA(\approx 1 \times$ $\left.10^{6} \mathrm{~K}\right)$. The raster data was binned by two along the 143 pixel slit slit resulting in 72 pixels of size $3.36^{\prime \prime}$ in $Y$. By rastering the $4^{\prime \prime}$ wide slit 60 times, raster images of size $\approx 240^{\prime \prime} \times 240^{\prime \prime}$ were built up. Both datasets were calibrated following the most up-to-date standard CDS calibration techniques ${ }^{2}$. All velocities in this work are relative line-of-sight (LOS) velocities measured relative to an "averaged" reference line profile, obtained by summing together all the individual lines at each pixel position along the slit (70) and at each time frame (85). For each of the 85 time frames in $\mathrm{s} 27152 \mathrm{r} 01$, the exposure time was $15 \mathrm{~s}$ leading to a cadence of $\approx 21 \mathrm{~s}$, a frequency resolution of $\approx 0.56 \mathrm{mHz}$ and a Nyquist frequency of $\approx 24 \mathrm{mHz}$.

\section{Results}

In Fig. 1 we show raster images (from dataset s27153r00) in different temperature lines as marked. The vertical lines show the location of the CDS slit, corresponding to the temporal series

\footnotetext{
${ }^{1}$ http://perswww.kuleuven.ac.be/ u0005791/werk/ EITshutt/jop.html

${ }^{2}$ http://solar.bnsc.rl.ac.uk/software/uguide/ uguide. shtml
}

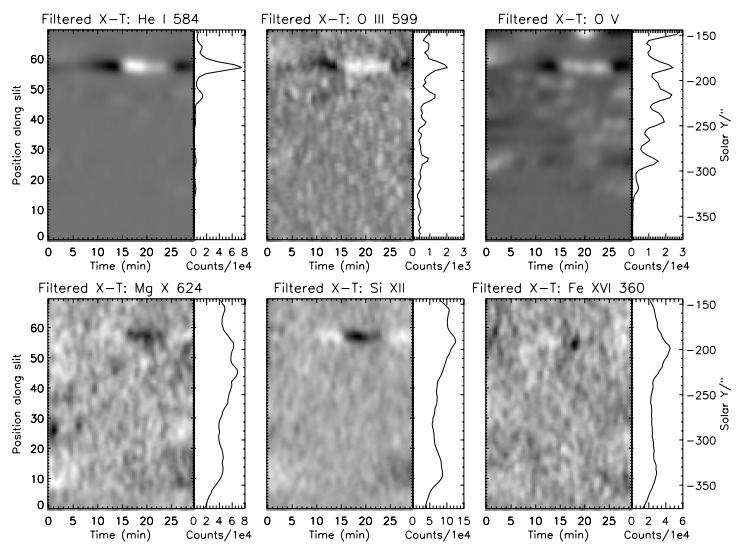

Fig. 2. Space-time behaviour of the intensity in the different temperature lines. The right panels show the summed (over time) count values.

dataset s27152r01 and the small white boxes within them mark the location of pixels 55-59, i.e., the pixels of interest along the CDS slit. As these raster images were obtained $\approx 50 \mathrm{~min}$ after the end time of the s27152r01 dataset, they are not very close in time to the time series data, which show dynamic short-lived events (which will be discussed later). However, note that in the raster images pixels 55-59 pass through a system of off-limb loop like structures only seen in the lower temperature He I $584.33 \AA$, O III $599.59 \AA$, O v $629.73 \AA$ lines, i.e., those formed at temperatures lower than $2.5 \times 10^{5} \mathrm{~K}$.

In Fig. 2 we show the space-time behaviour of the intensity (radiant flux) along the slit as recorded in the different temperature lines. The solar north-south (SOLAR_Y) direction is on the vertical axis and the horizontal axis is time. To bring out the details of the original intensity map we have filtered out the very bright low frequency components $(<0.3 \mathrm{mHz})$ in this image. Note that the white patches (intensity enhancements) in the top panels (corresponding to lower temperature lines) between $Y$-pixels 55-59 correspond to black patches (evacuation) in the lower panels (corresponding to coronal lines). We note that because this dynamic activity occurs within a short pixel range of 55-59 pixels this indicates that the "coherence length" of whatever is occurring is only 5 pixels or $\approx 17$ " long. The total number of counts in a pixel (summed counts) during the observation is shown in the right columns. In Fig. 3 we plot, against time, the variation of intensity (bold lines) and velocity (grey lines with error bars) corresponding to pixel 57, as recorded by different temperature lines. Locations where the Signal-to-Noise $(S / N)$ dropped below a value of 5 were not plotted. For this reason the results for O III are not shown as its $S / N$ was consistently below a value of 5 . We should point out that the velocities shown in this figure are projected (relative) velocities onto the line-ofsight, not actual velocities, and that the "real" velocities may, in fact, be larger than those shown. Large enhancements in intensity for He I and $\mathrm{O} \mathrm{V}$ are recorded in the data. These enhancements occur in association with unusually strong blue-shifts of up to $100 \mathrm{~km} \mathrm{~s}^{-1}$, which do not, however, reach their maximum values until after the intensity has once again diminished to its pre-enhancement levels. It is noticeable, for example, that in He I the strong blueshifts of $\approx 100 \mathrm{~km} \mathrm{~s}^{-1}$ occur both before and some time after the main increase in intensity. During the enhancement itself there is initially a small trend towards the red. In $\mathrm{OV}$ there is a progressive increase in blueshifted velocity as the brightening diminishes, reaching a maximum when the intensity has decreased to pre-enhanced levels. However, then, contrary to 

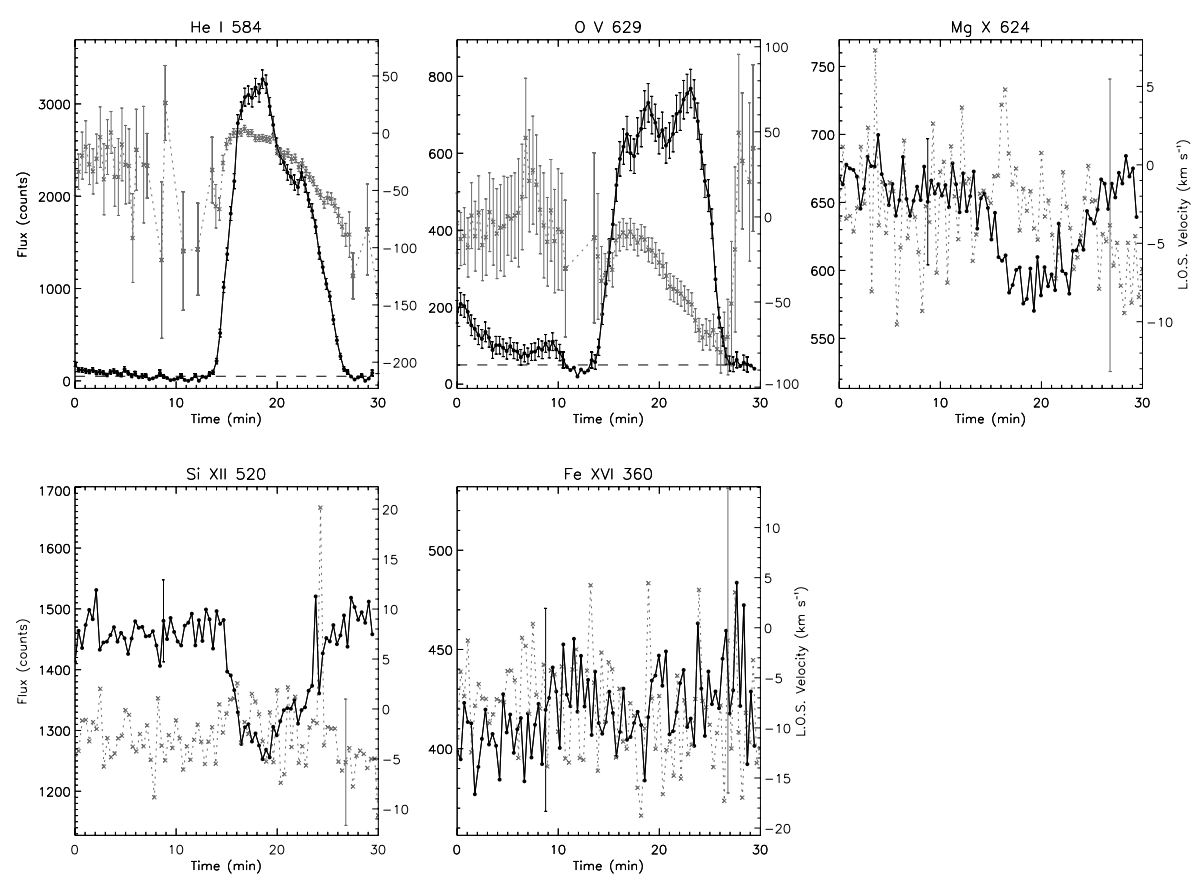

Fig. 3. Variation with time of intensity (black lines) and velocity (grey lines) corresponding to pixel 57 of dataset s27152r01. The horizontal dashed lines indicates the Signal-to-Noise level $(S / N)$ of 5 . O III is not shown due to its low count values $<S / N=5$.

what happens in He I, there is a rapid change and an increase to redshifted velocities from a time of $\approx 27 \mathrm{~min}$ onwards. It is not clear what the reason for these large velocity jumps in He I and $\mathrm{OV}$ is, although we can speculate that the constantly increasing blueshift velocities, occuring during a time when the intensity is enhanced, and which reach a maximum when the intensity enhancement has come to an end, are a response to downfalling, perhaps, accelerating, plasma from the corona impacting on the higher densities of the TR. This would tally with the decreases in intensity seen in the coronal lines. This interpretation has some support in the theory, in that Müller et al. (2005) found that simulated fast blobs can decelerate and "bounce" at the transition region, with a resulting change in velocity sign. This would agree well with what we found in the $\mathrm{OV}$ line even down to the fact that the rebound velocity (in our case redshifts of $40-50 \mathrm{~km} \mathrm{~s}^{-1}$ from a time of $\approx 27$ min onwards) is less than the initial downflow speed (some $\approx 90 \mathrm{~km} \mathrm{~s}^{-1}$ ). In the coronal lines we do not see any significant velocity-shifts, only decreases in the intensities of the $\mathrm{Mg} \mathrm{X}$ and $\mathrm{Si}$ XII lines at the times the cooler lines show enhancements. There is, however, a spike in the Si XII velocity to a redshift of $\approx 20 \mathrm{~km} \mathrm{~s}^{-1}$ at the approximate point at which $\mathrm{OV}$ shows its maximum blueshift. We have examined the line profile of this particular event and confirmed that this behaviour is real and not due to a bad fit, etc. In fact, we find the same behaviour at this same time, in the same line, in the neighbouring pixel, No. 56. While it is curious that we do not see a similar behaviour in the other coronal lines, this change from a redshift in the coronal lines to a blueshift in TR lines could suggest an inflow from the coronal lines to the lower temperature TR lines.

To investigate what may be occurring, we have analysed the TRACE data from the day. This was only available in the $171 \AA$ filter at around $1 \times 10^{6} \mathrm{~K}$, so at the same temperature as the Mg IX line in the rasters. From the TRACE data we have produced a movie ${ }^{3}$. From this movie, it can be seen that between the times of the enhancement (in the cooler CDS lines), $\approx 10: 45-10: 59$ UTC, there is a loop draining (or perhaps a Propagating Disturbance (PD)) at roughly $X=950-970, Y=$ $-180 \rightarrow-160$. This dynamic behaviour is most pronounced

\footnotetext{
${ }^{3}$ See http://star.arm.ac.uk/preprints/
}

between $\approx 10: 53$ and 10:59 UTC (when the TRACE movie ends). The location of the CDS slit in these trace images/movies can be seen from the thick white lines, where again the selected pixels 55-59 are highlighted as white boxes. The location of the CDS slit was obtained by cross-correlating the CDS raster image of Mg IX with a similarly sized TRACE 171 subset image, covering the same field of view. While the CDS slit does not pass through any of the coronal emission seen with TRACE 171, we know from Fig. 1 that at TR temperatures the slit passes through cooler loops. We can therefore speculate that what we see in our results here, the brightenings in the cooler TR lines and darkenings in the coronal lines, may be due to plasma motions taking place in a loop or loops.

It was noticed when looking at the variation of intensity in Fig. 3 that there was evidence of oscillations in the enhanced part of the $\mathrm{OV}$ intensity. To explore if these apparent oscillations were real and also present in other lines, we performed a time series analysis, following O'Shea et al. (2007), on all our TR and coronal lines. It was found that only the He I and $\mathrm{O} \mathrm{V}$ lines possessed statistically significant oscillations. The results of this analysis are shown in Figs. 4 and 5 for He I and O V, respectively. We note that the intensities (radiant fluxes) shown are relative intensities, produced by dividing the original time series by the slowly varying background and then subtracting a value of 1 from the result. The background is calculated as being any frequency below that corresponding to one third of the total length of the time series (i.e. any oscillations with less than three repetitions are not considered "real"). This meant, in practice, that we calculated the background by lo-pass filtering everything below $\approx 1.7 \mathrm{mHz}$. In the case of the He I line in Fig. 4 , a frequency of $2.83 \mathrm{mHz}$ is found, together with what appears to be its harmonic at $5.10 \mathrm{mHz}$ (given the frequency resolution of $0.56 \mathrm{mHz}$ ). Both frequencies are above the statistically significant $95 \%$ probability. In the case of the OV line (Fig. 5), a strong frequency of $2.83 \mathrm{mHz}$ is again found, together with its (non-statistically significant) harmonic at $5.10 \mathrm{mHz}$. The presence of these harmonic frequencies, at frequencies similar to those found by (O'Shea et al. 2007), suggests that the intensity enhancements/decreases we observe are occurring within a loop 

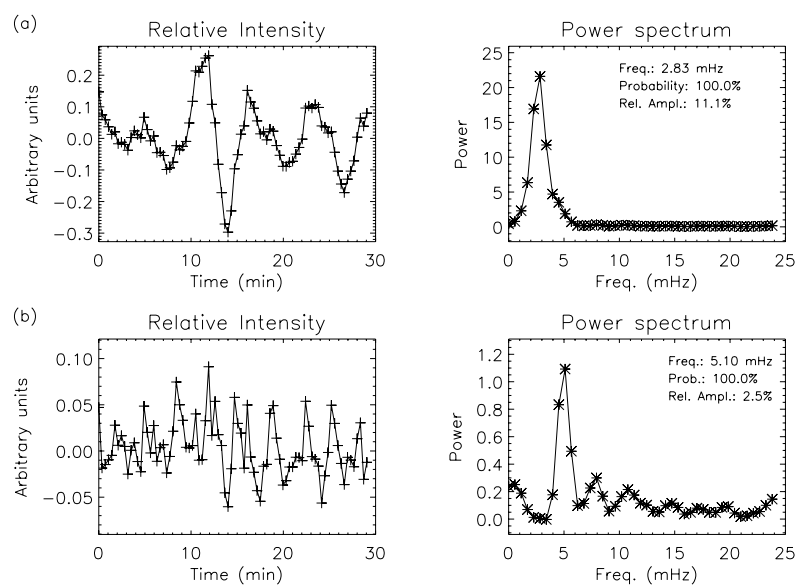

Fig. 4. a) (Left panel) Time series of the relative intensity in He I; (right panel) the resulting power spectrum. b) Pre-whitened time series and the resulting power spectrum.
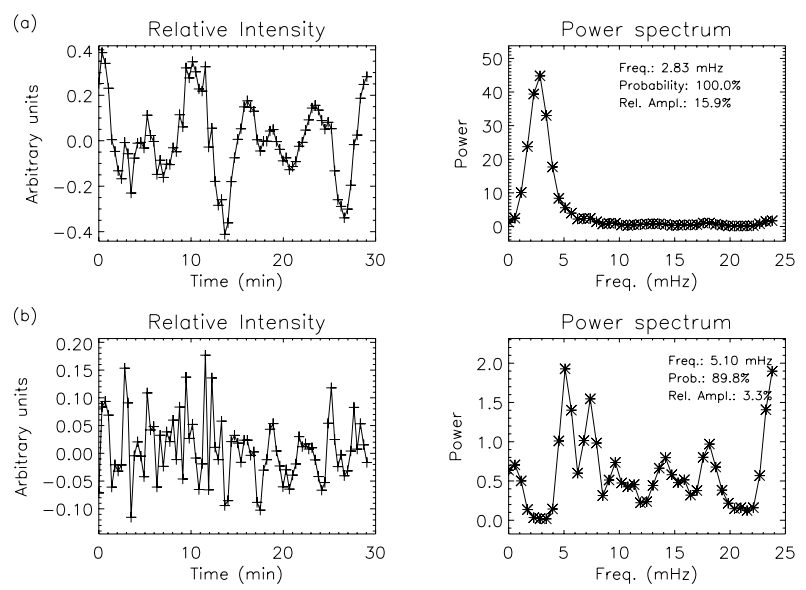

Fig. 5. a) Time series and power spectrum for O v. b) Pre-whitened time series and resulting power spectrum.

or loops in which standing waves and, perhaps, propagating disturbances (PDs), are present. Support for this comes in the form of Fig. 1 where loops are seen to cross the CDS slit in the raster images of He I and O V. Following on from the conclusions of O'Shea et al. (2007) we may also suggest that any waves present in the loops may be fast kink waves. We note, however, that O'Shea et al. (2007) found no evidence of decreases in intensity at coronal temperatures, suggesting that their situation and the one described here may not be entirely the same.

\section{Discussion and conclusions}

In this work, simultaneous observations with CDS and TRACE have been used to investigate plasma condensation processes in active region loops observed at the solar limb. Through the use of spectroscopic diagnostics, we have found large velocity shifts and sudden intensity enhancements in TR lines that are mirrored by decreases in the intensity of coronal lines. The CDS temporal sequence used allowed us to detect this dynamic behaviour simultaneously in different temperature lines and, for the first time (to our knowledge), to study it with a time series analysis. For earlier studies of active region loops dynamics, only CDS raster sequences have been used (Fredvik et al. 2002; Kjeldseth-Moe $\&$ Brekke 1998). The intensity enhancements found by us are probably the result of plasma condensation: hot coronal plasma is cooled to TR or even chromospheric temperatures and slides down the loop legs at speeds of up to $100 \mathrm{~km} \mathrm{~s}^{-1}$. This process has also been known in the literature as coronal rain. We should also point out here that we looked for GOES X-ray and LASCO data in an effort to find any possible link with flares or CMEs, but during the time period of our observations no major flares and/or CMEs were detected. This leads to a deeper question, what causes these plasma condensations? Recently Müller et al. (2005) presented a comparison of observed intensity enhancements from an EIT shutterless campaign with non-equilibrium ionization simulations of coronal loops in order to reveal the physical processes governing fast flows and localized brightenings. They show that catastrophic cooling around the loop apex as a consequence of footpoint-concentrated heating can perhaps offer a simple explanation for these observations. Along with plasma condensation, we find the presence of harmonics in the intensity oscillations which indicates the existence of waves within the coronal loops.

Acknowledgements. We thank the CDS and TRACE teams for their help in obtaining these data. CDS is a part of SOHO, the Solar and Heliospheric Observatory, a project of international cooperation between ESA and NASA. This work was supported in part by a PRTLI research grant for Gridenabled Computational Physics of Natural Phenomena (Cosmogrid) and PPARC grant PP/D001129/1. We thank the Royal Society and the British Council for funding visits between Armagh Observatory and the Indian Institute of Astrophysics.

\section{References}

De Groof, A., Berghmans, D., van Driel-Gesztelyi, L., \& Poedts, S. 2004, A\&A, 415,1141

De Groof, A., Bastiaensen, C., Müller, D. A. N., Berghmans, D., \& Poedts, S. 2005, A\&A, 443, 319

Doyle, J. G., Taroyan, Y., Ishak, B., Madjarska, M. S., \& Bradshaw, S. J. 2006, A\&A, 452, 1075

Fredvik, T., Kjeldseth-Moe, O., Haugan, S. V. H., et al. 2002, Adv. Space Res., 30,635

Handy, B. N., Acton, L. W., Kankelborg, C. C., et al. 1999, Sol. Phys., 187, 229 Harrison, R. A., Sawyer, E. C., Carter, M. K., et al. 1995, Sol. Phys., 162, 233 Kjeldseth-Moe, O., \& Brekke, P. 1998, Sol. Phys., 182, 73

Levine, R. H., \& Withbroe, G. L. 1977, Sol. Phys., 51, 83

Müller, D. A. N., de Groof, A., Hansteen, V. H., \& Peter, H. 2005, A\&A, 436, 1067

O’Shea, E., Srivastava, A., Doyle, J., \& Banerjee, D. 2007, A\&A, 473, L13

Schrijver, C. J. 2001, Sol. Phys., 198, 325

Teriaca, L., Banerjee, D., Falchi, A., Doyle, J. G., \& Madjarska, M. S. 2004, A\&A, 427, 1065 